apocalypse on science. The core of the threat is not only science but also knowledge. The book rings the alarm but provides no answers because there are none. Scientists are motivated by curiosity and a yearning to understand nature, but the real engines of science are business and war. Without global cultural revolution, business, war, science and education will surely continue. Can we manage the dark side of science and prove Rees wrong? Only time will tell.

I highly recommend this provocative and educational book. It is written for broad appeal and even includes a crowd-pleasing array of references to popular culture. If we do survive to see the twenty-second century, I hope that Martin Rees' cautionary and alarmist book will be required high-school reading, a twenty-first-century analogue of Rachel Carson's Silent Spring. I also recommend Future Evolution by Ward and Alexis Rockman (reviewed in Nature 416, 125-126; 2002), a discussion of the fate of humans that favours their survival but warns of dire effects on the rest of the planet's inventory of plants and animals.

Don Brownlee is in the Department of Astronomy, University of Washington, Seattle, Washington 98195, USA.

\section{Four legs bad, two legs good}

\section{Lowly Origin: Where, When, and Why Our Ancestors First Stood Up by Jonathan Kingdon}

Princeton University Press: 2003. 408 pp.

$\$ 35, \mathfrak{2} 24.95$

\section{Lorenzo Rook}

Jonathan Kingdon is not only a zoologist with a deep knowledge of African mammals, but also a fine narrator and a talented artist, so any new book by him is particularly welcome. In Lowly Origin he offers the reader a finely illustrated book on human evolution, focusing particularly on the transition from four-legged apes to two-legged hominins.

The origin of our ability to walk on two legs is one of the most debated subjects in anthropology. Here Kingdon attempts to explain to a wide audience, and to shed new light on, the origin and consequences of this turning point in human evolution. The book's strength is its broad perspective, bringing ecological, palaeoenvironmental and palaeogeographical evidence into an analysis of our "lowly origin" (the title is taken from the last two words of Darwin's The Descent of Man).

Over ten chapters, Kingdon provides views on the evolutionary trajectories of forelimbs since Palaeozoic tetrapods, ponders the

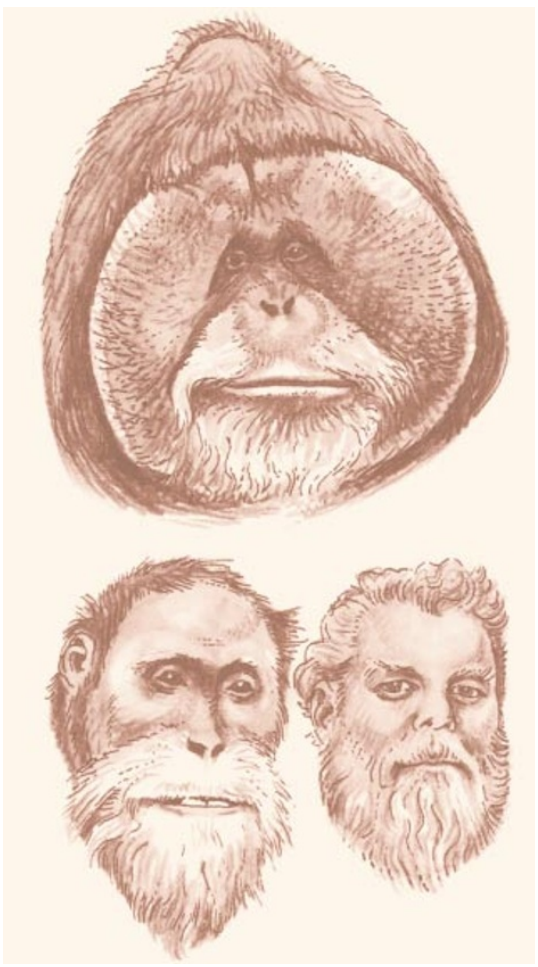

Face facts: Jonathan Kingdon highlights the similar expressions of humans and orang-utans.

evolutionary patterns of African hominins as driven by physical limits between geographic regions (by 'basin evolution'), and discusses the role of brain expansion in driving the modern diaspora. He repeatedly refers to "self-portraits", both as an abstract mental exercise comparing humans and other primates or animals, and by including illustrations of himself in comparison with other primates and humans - the example shown above is just one of many intriguing juxtapositions. As a "repentant vandal", Kingdon summarizes in the final chapter our status as the last survivors of the many bipedal African mammals that persisted until recent times, and offers insight into the future of our species.

There are a few weak points, however. In some places the ongoing debate on some crucial issues is missing and a definite position is taken instead. For example, the recently described Sahelanthropus fossil from Chad is mentioned as a gorilla-like ape but is totally overlooked in the chapters that discuss the earliest hominin.

Another case is the origin of the African ape-human clade (the taxonomic group including Homo and his nearest living relatives, Pan and Gorilla). Some recent papers have argued that the European Late Miocene apes were the ancestors of the African ape-human clade, suggesting that hominids left Africa for Europe about 20 million years ago and returned some 10 million years later. Kingdon accepts this 'out of Africa and back' hypothesis - the Eurasian origin of his "squatting ground ape" is a recurrent motif throughout the first part of the book. But in doing so, he neglects the wide palaeoecological perspective that is otherwise the main strength of this book.

The European hominoid with the widest temporal and geographical range is Dryopithecus, a large-bodied arboreal ape adapted for a diet of soft fruits. A slightly more recent European ape is Ouranopithecus (also known as Graecopithecus), a hominoid about the size of a female gorilla that is adapted to eating hard fruits. Dryopithecus is associated with swampy subtropical environments (from Spain to Eastern Europe), whereas Ouranopithecus (from Greece) and close relatives from Turkey lived in rather younger, more open woodland.

Does the available palaeontological and palaeoecological information support the dispersal of Dryopithecus or Ouranopithecuslike forms from Europe into Africa? The 'out of Africa and back' hypothesis is essentially based on 'strong' cladistic analyses (a procedure for organizing the evolutionary relationships among taxa on the basis of shared anatomical characters) that represent Dryopithecus and Ouranopithecus as being sister taxa of the African ape-human clade. This hypothesis is favoured by some authors because there was a 'hominoid vacuum' in Africa between 12.5 million and 6 million years ago. But the large-mammal fossil record in Africa is relatively poor for this time interval, so the absence of hominoid fossils cannot be taken as evidence that hominoids were not living in this area during this period.

Dryopithecus was dependent on equable, subtropical temperate forests, below-branch locomotion and a soft-fruit diet, so it is not likely to have moved across the more open woodland of the southwestern Mediterranean during the Late Miocene. Ouranopithecus is also an unlikely ancestor of the African ape-human clade, because its teeth and jaws had thick dental enamel and were adapted for a diet of hard fruits and seeds. As such they were more comparable to the teeth of later mid-Pliocene to early Pleistocene hominids (between 3 million and 1 million years ago) than to the thin-enamelled teeth of the early bipedal hominin of Ethiopia or the earliest Late Miocene hominin from Kenya or Chad.

Despite its lack of discussion of these issues, Lowly Origin should interest a wide audience. It will serve as supplementary reading for researchers and graduates, but should also be accessible to lay readers. Kingdon is an authority on Africa's zoology, and has merged his deep knowledge of mammal evolution with his love for Africa and art.

Lorenzo Rook is in the Department of Earth Sciences and the Natural History Museum, University of Florence, via G. La Pira 4, 50121 Firenze, Italy. 